\title{
THE DOUBLE NUCLEUS GALAXIES MKN 423 AND MKN 739
}

\author{
Piero Rafanelli \\ Department of Astronomy, University of Padova, Italy \\ Paolo Marziani \\ International School for Advanced Studies, Trieste, Italy
}

1. Mkn 423

\section{1a. Introduction}

Long slit spectroscopy and imaging of Mkn 423 and Mkn 739 have been performed at the $2.2 \mathrm{~m}$ and $3.5 \mathrm{~m}$ telescopes of the Calar Alto Observatory using both CCD and photographic detectors. Low and medium resolution spectra $(1.8 \AA, 3.5 \AA, 6.0 \AA)$ together with $\mathrm{V}$ images have permitted to demonstrate the merging nature of Mkn 423 and the double structure of the NLR of its Seyfert component. This last feature has been found also in the NLR of the Seyfert component of Mkn 739, a double system the two components of which are counterrotating.

\section{1b. Morphology}

Mkn 423 is a Seyfert galaxy located in the Abell cluster No. 1257 at an angular distance of 10 arcmin from its center, which at the redshift of the galaxy $z=0.0325$ corresponds to a linear distance of $0.28 / h \mathrm{Mpc} \quad($ scale $=0.47 / \mathrm{h} \mathrm{kpc} / \mathrm{arcsec})$. The complex nature of this galaxy was firstly recognized by Markarian and Lipovetskii (1974), who described Mkn 423 as a spherical galaxy with a jet. More recently Dahari (1985) classified this object as a double nucleus galaxy with distorted morphology in his survey of Seyfert galaxies with companions. The contour map of a deep image, Figure 1, shows the presence of a resolved structure of elliptical shape $B$, located at a distance $d=9 \operatorname{arcsec}\left(P . A .=169^{\circ}\right)$ from the bright Seyfert nucleus $A$. The major and minor axis of $B$ have angular size $a=4.5$ arcsec, $b=3$ arcsec respectively and the P.A. of $\mathrm{a}$ is $188^{\circ}$. The Seyfert nucleus $\mathrm{A}$ has spherical symmetry and its radius is 5 arcsec. $A$ and $B$ are connected by a bridge of matter and are both surrounded by a pear-shaped structure of size $30 \times 19$ arcsec.

\section{1c. Emission-line regions}

Long slit spectra obtained setting the slit in the N-S direction along the Seyfert nucleus $A$ and the region $B$ show strong emission lines of [SII], [NII], $\mathrm{H} \alpha$ and $\mathrm{H} \beta$, which extend on the southern side of the spectrum of $\mathrm{A}$. $\mathrm{H} \alpha$ peaks in correspondence of $\mathrm{B}$ and lengthens over the southern part of the galaxy indicated with $\mathrm{C}$ in Figure 2. Conversely the [OIII] nebular lines are not extended, but confined to the spectrum of $A$. The contour map of the [OIII] $\lambda 5007$ nebular line, Figure 3, shows that the core of the line is formed by two knots $A 1$ and $A 2$ of different heliocentric radial velocity, $v_{A 1}=9580 \mathrm{~km} / \mathrm{s}, \mathrm{v}_{A 2}=9760 \mathrm{~km} / \mathrm{s}$, from which two components stream out each up to a distance of 5 arcsec from the nucleus: one from A1 towards southern,the other one from A2 towards northern. We take $v_{A 2}$ as the systemic velocity. The centers of $A 1$ and $A 2$ are not aligned along the direction of the dispersion, Figure 3, A2 being located $\leq 1.0$ arcsec to the northern of A1. However the spatial separation along the slit of the two nuclear blobs is not large enough to extract their spectra.

The three regions $\mathrm{A}, \mathrm{B}$ and $\mathrm{C}$, indicated in Figures 1, 2, have been then used for the diagnostics of the emitting gas. The spectrum of the nucleus $A$ is characterized by the presence beneath $\mathrm{H} \alpha$ of a broad component, not present in the $\mathrm{H} \beta$ profile, as it is common in Seyfert 1.9 galaxies.

The [OIII] $\lambda 5007 / \mathrm{H} \beta$ versus $[\mathrm{SII}] \lambda \lambda(6716+6731) / \mathrm{H} \alpha$ and $[\mathrm{OIII}] \lambda 5007 / \mathrm{H} \beta$ versus $[\mathrm{NII}] \lambda$ $6583 / \mathrm{H} \alpha$ diagnostic diagrams, Figures $4 \mathrm{a}, \mathrm{b}$, (Veilleux and Osterbrock, 1987) indicate that the Narrow Line Region of $\mathrm{A}$ is produced by gas photoionized by a non thermal source, while $\mathrm{B}$ and $\mathrm{C}$ are both low excitation HII regions. The FWHMs of the narrow lines are 
significant for the Seyfert nucleus A only (FWHM=400 $\pm 64 \mathrm{~km} / \mathrm{s}$ ) since they are typical of the instrumental profile for the $B$ and $C$ regions.

The narrow component of $\mathrm{H} \alpha$ in the spectrum of $\mathrm{A}$ has luminosity $\mathrm{L}(\mathrm{H} \alpha)_{A}=3.34 \times 10^{40} /$ $h^{2} \mathrm{erg} / \mathrm{s}$ (Osterbrock, 1981). It follows that $\mathrm{L}(\mathrm{H} \alpha)_{B}=2.25 \times 10^{40} / h^{2} \mathrm{erg} / \mathrm{s}, \mathrm{L}(\mathrm{H} \alpha)_{C}$ $=0.40 \times 10^{40} / h^{2} \mathrm{erg} / \mathrm{s}$ which correspond to a number of ionizing photons $\mathrm{Q}(\mathrm{H})_{B}=4.75$ $\times 10^{52} / h^{2} / s, \mathrm{Q}(\mathrm{H})_{c}=8.44 \times 10^{51} / h^{2} / s$. A comparison of the $\mathrm{H} \alpha$ luminosities of $\mathrm{B}$ and $\mathrm{C}$ with those typical of Hot Spots, Disk HII Regions, Starburst Nuclei and HII Nuclei (Kennicut et al., 1989) shows that C can be classified as a Disk HII Region, while B can be either one of the most luminous known Disk HII Regions or a Starburst Nucleus.

\section{1d. Kinematics}

The velocity curve of $\mathrm{Mkn} 423$ is shown in Figure 5 where we have indicated the position and the size of the emitting regions $A, B$ and $C$. It is evident that $A$ and $B$ are two counterrotating systems since their rotation curves have an opposite inclination. In addition the systemic velocity of $B$ is $100 \mathrm{~km} / \mathrm{s}$ smaller than the systemic velocity of $A$ suggesting that $A$ and $B$ could be two independent, very close systems. The velocity curve of $C$ is the continuation of the southern branch of the velocity curve of $B$ and keeps its inclination. The same behavior is shown by the velocity curve between $A$ and $B$. This suggests that $C$ and the region between $A$ and $B$ belong to $B$ itself as it is further supported by their comparable size and by their symmetric position relative to the center of $B$.

\section{1e. Discussion}

The data collected in this work lead to some interesting conclusion on the complex nature of Mkn 423. The presence of two emitting blobs A1 and A2 in the Seyfert nucleus A reminds the results obtained by Whittle et al. (1988) in their study of 'Radio lobes and [OIII] profile sub-structure in Seyfert galaxies' where they found that emission line gas is closely associated with individual radio lobes. This strongly suggests that high resolution radio mapping of the nucleus of Mkn 423 should reveal the presence of at least two radio lobes in the position of A1 and A2 respectively. Many of our results hint the idea that the $B$ region is a galaxy which is merging with the Seyfert nucleus. The morphology and the size of B, its $\mathrm{H} \alpha$ luminosity, typical of a Starburst nucleus, and finally its kinematical independency from the Seyfert nucleus are all evidences that support the classification of $B$ as a galaxy and then the merging nature of Mkn423.

\section{2. $M k n 799$}

\section{2a: Morphology}

Mkn 739 was firstly recognized as a double nucleus galaxy by Petrosian et al. (1979). Its two stellar components, a Seyfert nucleus $A$ and a compact emission line region B, Figure 6, are aligned along the east-west direction and their angular separation is $\sim 6$ arcsec, which at the redshift of the galaxy $z=0.0300$ corresponds to $\sim 2.64 h^{-1} k p c$ (Scale $=0.44 h^{-1} k p c$ ). The two nuclei are located close to the center of a nearly spherical envelope of radius $\sim$ 20 arcsec which shows a slight deformation towards northern on its upper side.

\section{2b. Emission line regions}

Long slit spectra, obtained setting the slit in the east-west direction along the two nuclei $A$ and $B$, permit to isolate 4 emitting line regions: the two nuclei $A$ and $B$ and two regions $C$ and $D$ located on the western side of $B$ and on the eastern side of $A$ respectively, Figure 6. The contour map of the spectral region $\mathrm{H} \beta-[\mathrm{OIII}] \lambda 5007$, Figure 7 , gives the size and the location of the regions $A, B, C$ and $D$. It is worth noting that the spectrum of $D$, Figure 7 , is characterized by the lack of any trace of $\mathrm{H} \beta$ and by a strong [OIII] $\lambda 5007$ line, conversely the spectra of $B$ and $C$ show either lines. Since the [OIII] $\lambda 5007 / \mathrm{H} \beta$ ratio is an indicator of the ionization degree, it is evident that this is higher in $D$ than in $B$ and $C$. Another interesting feature is the presence of two blobs $A 1$ and $A 2$ of different radial velocity $v_{A 1}=8870 \mathrm{~km} / \mathrm{s}$ 


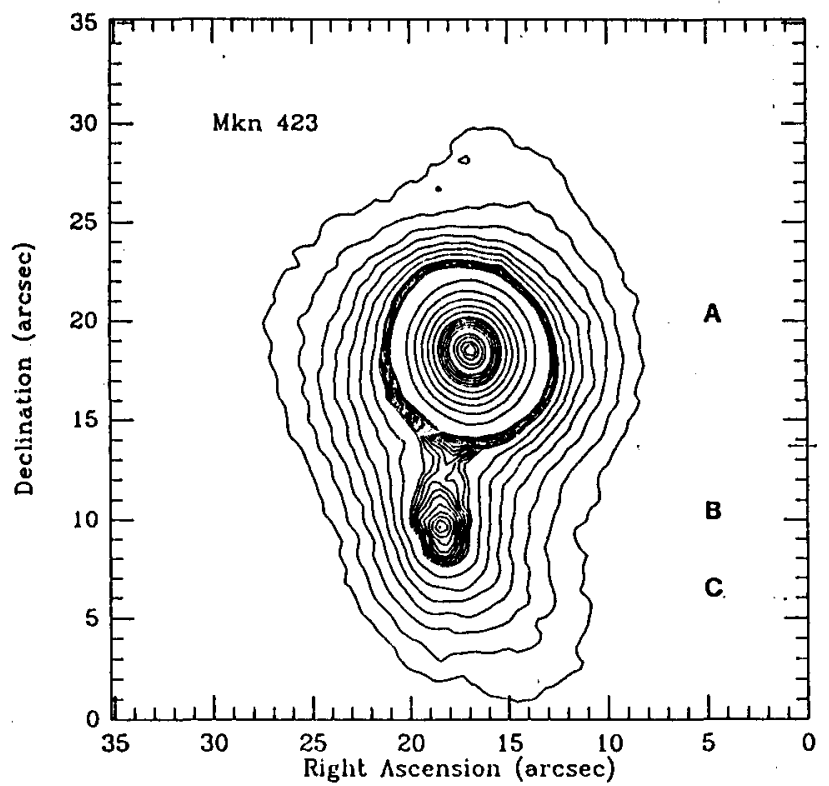

FIG. 1

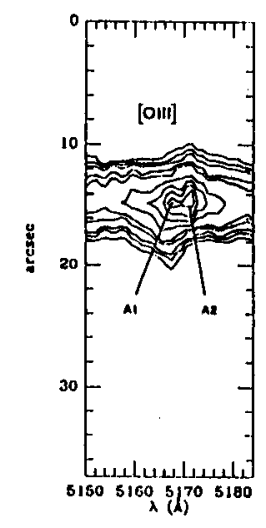

FIG.3

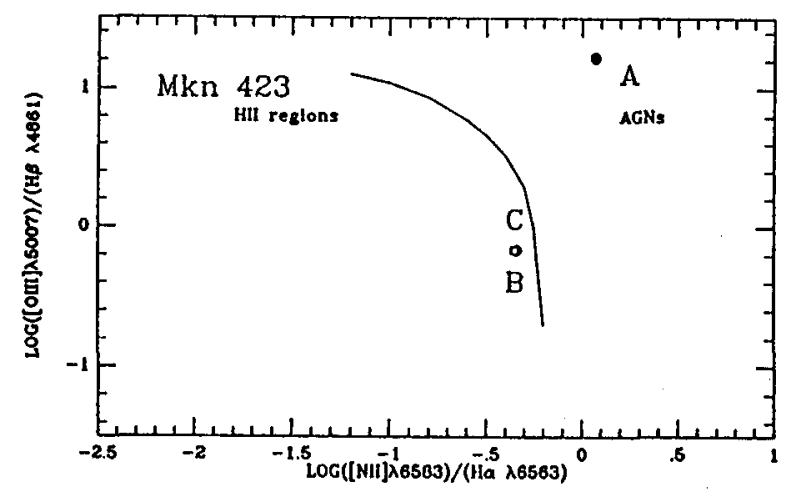

FIG. 4b
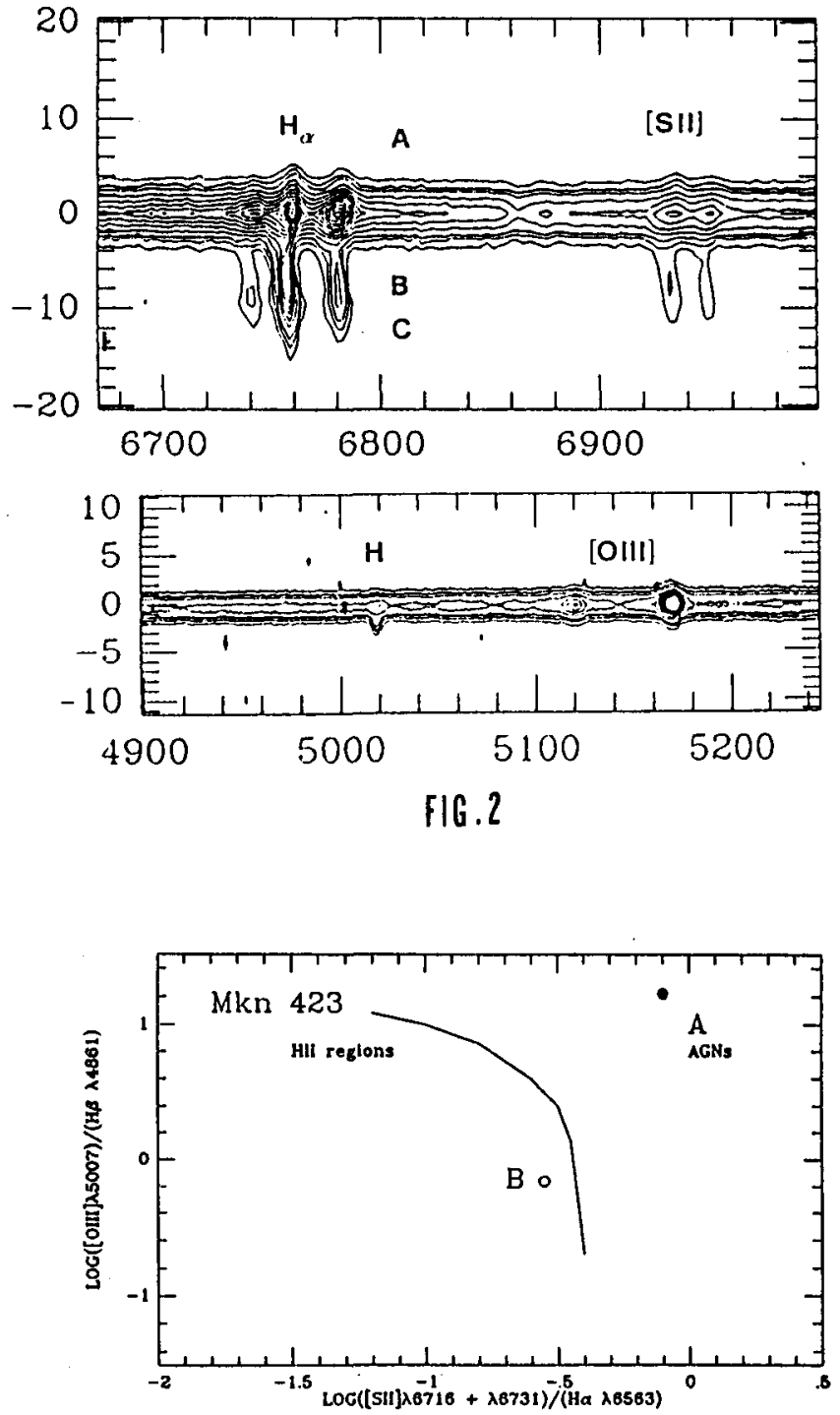

FIG.4a

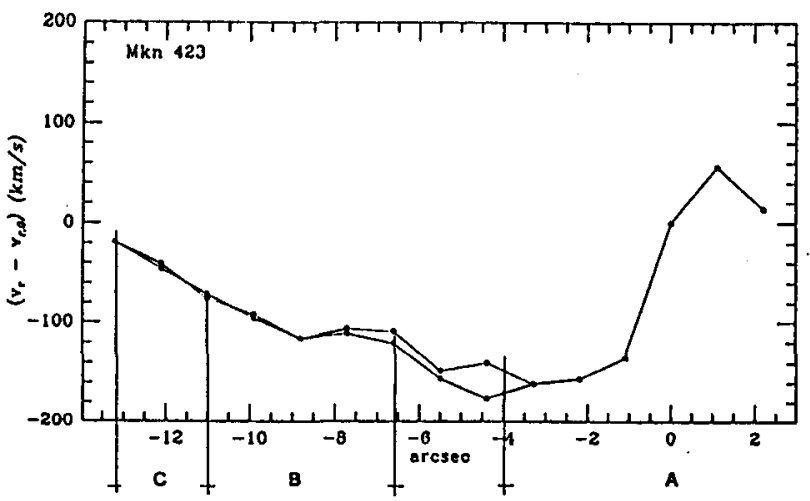

FIG.5 


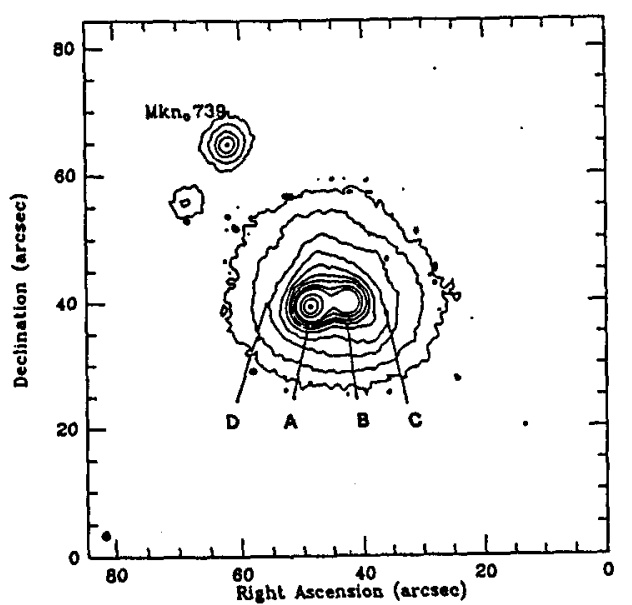

FIG.6

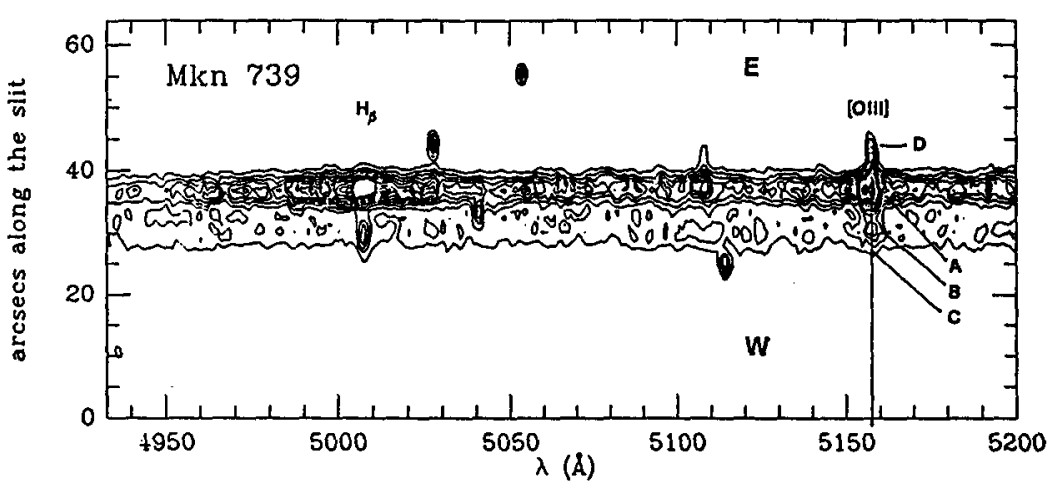

FIG.7

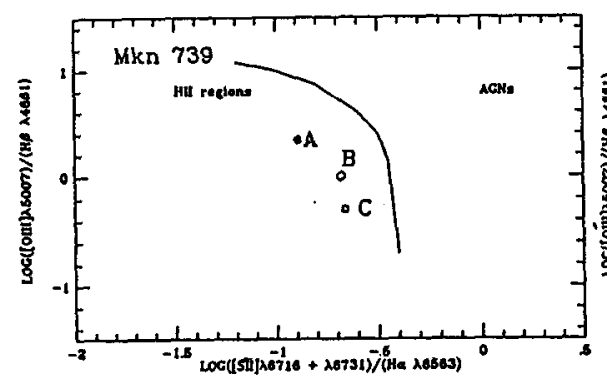

FIG. $8 \mathrm{a}$

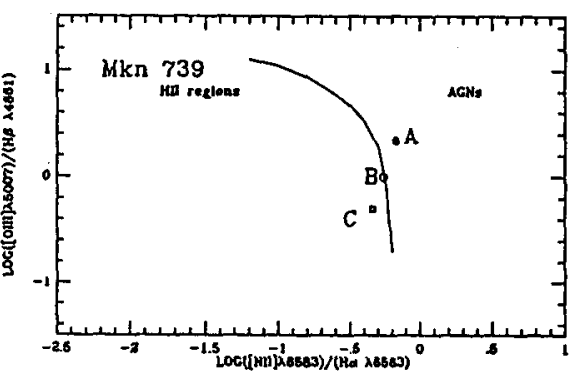

FIG.8b

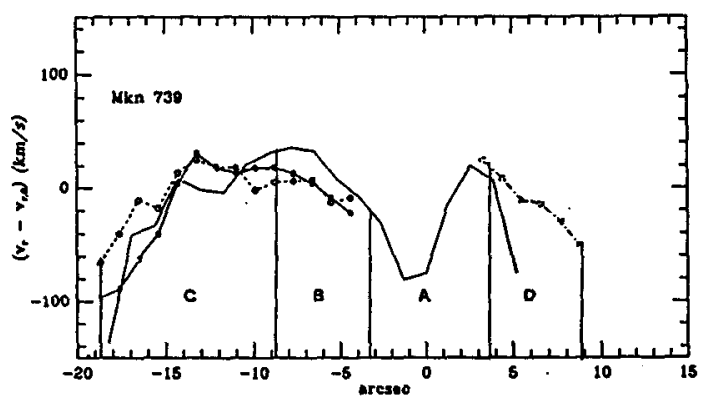

FIG.9

$\mathrm{v}_{A 2}=9020 \mathrm{~km} / \mathrm{s}$ in the core of the line [OIII] $\lambda 5007$, Figure 7, emitted by the Seyfert nucleus A. The two blobs are located along the direction of the dispersion (east-west) and are not spatially separated along north-south. It is evident from Figure 7 that the systemic velocity of $\mathrm{B}, \mathrm{C}$ and $\mathrm{D}$ corresponds to that of $\mathrm{A} 2$ as it is confirmed by the position of their $\mathrm{H} \alpha$ and [NII] lines. We take $\mathbf{v}_{A 2}$ as the systemic velocity of Mkn 739 .

The two blobs A1 and A2 can be considered as the optical counterpart of the two radio lobes observed at $6 \mathrm{~cm}$ and $20 \mathrm{~cm}$ by Netzer et al. (1981) in the nucleus A of Mkn 739 . Their position along the north-south direction and their small separation $\leq 0.3 \mathrm{arcsec}$ in the radio maps are consistent with their lack of separation in the optical. The FWHM of the narrow lines in the spectrum of the Seyfert nucleus $\mathrm{A}$ is $\sim 290 \mathrm{~km} / \mathrm{s}$, conversely all spectral lines of $B, C$ and $D$ have an instrumental profile. However the profile of [OIII] $\lambda 5007$ in the spectrum of the Seyfert nucleus is composite. It shows two peaks which correspond to the two blobs observed in the contour map of the line and an underlying semibroad component with FWZI 2900 $\mathrm{km} / \mathrm{s}$. 
The diagnostic diagrammes of of Veilleux and Osterbrock (1987) applied to the line ratios of B, C, D and to those of the narrow component of $\mathrm{A}$ show, Figures $8 \mathrm{a}, \mathrm{b}$, that $\mathrm{B}$ and $\mathrm{C}$ are typical low excitation HII regions, that $\mathrm{D}$ is ionized by a nonthermal source ([OIII $] \lambda 5007 / \mathrm{H} \hat{\beta} \geq 1.1)$ and that the NLR of $\mathrm{A}$ can be classified either as a HII region or as a region ionized by a nonthermal source. This ubiquity in the classification of $A$ can be explained in terms of an underestimate of the intensity of the [SII] lines. Since the [SII] doublet is partially plunged in the atmospheric absorption band, the [SII] $/ \mathrm{H} \alpha$ ratio is reduced and the point representing $A$ in Fig. $8 \mathrm{a}$ is moved towards the area typical of the HII regions. The luminosity of the narrow component of the $\mathrm{H} \alpha$ line emitted by the Seyfert nucleus $\mathrm{A}$ is $\mathrm{L}(\mathrm{H} \alpha)_{A}=7.14 \times 10^{40} / \mathrm{h}^{2} \mathrm{erg} / \mathrm{s}$ (Shuder and Osterbrock 1981). It follows that $\mathrm{L}(\mathrm{H} \alpha)_{B}=2.93 \times 10^{40} / h^{2} \mathrm{erg} / \mathrm{s}, \mathrm{L}(\mathrm{H} \alpha)_{C}=2.57 \times 10^{40} / h^{2} \mathrm{erg} / \mathrm{s}$, which correspond to a number of ionizing photons $\mathrm{Q}(\mathrm{H})_{B}=6.18 \times 10^{52} / h^{2}$ photons $/ s, \mathrm{Q}(\mathrm{H})_{C}=5.42 \times 10^{52} / h^{2}$ photons $/ s$.

The $\mathrm{H} \alpha$ luminosities of $\mathrm{B}$ and $\mathrm{C}$, the two regions which are ionized by thermal sources,

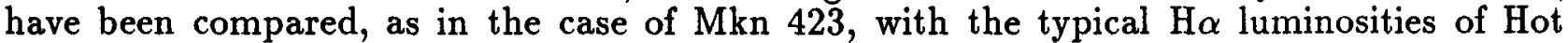
Spots, Disk HII Regions, Starburst Nuclei, HII Nuclei. It results that B can be classified as a Starburst Nucleus connected to a giant HII Region C.

\section{2c. Kinematics}

The velocity curve along the line joining the two nuclei is shown in Figure 9 . The velocity of the nucleus $B$ has been arbitrarily put equal to zero while the zero of the angular distances has been put in correspondence of the Seyfert nucleus A. The strange shape of the velocity curve within the nuclear region $A$ arises from the double structure of the core of the narrow lines like [OIII] $\lambda 5007$. In fact the different intensity and extension along the slit of the two components the line produces a typical $U$ displacement of the center of their blend along the normal to the dispersion. The extranuclear region $\mathrm{C}$ connected to the nucleus $\mathrm{B}$ and the region $\mathrm{D}$ connected to the nucleus $\mathrm{A}$ are characterized by a velocity distribution of opposite inclination which suggests counterrotation of the two nuclei and of their connected gas.

\section{2d. Discussion}

The results of this investigation can be summarized as follows:

1. The NLR of the Seyfert component $A$ is formed by two blobs of different radial velocity, likely due to rotation around their center of mass.

2. The eastern extranuclear region $D$ is ionized by the nonthermal nucleus $A$.

3. The two main components the system are counterrotating.

\section{References}

Dahari O.: 1985, Astron. J. 90, 1772

Kennicut R.C., et al. : 1989 Astron. J. 97, 1022)

Markarian B.E., Lipovetskii V.A.: 1974, Astrophysics 8, 89

Netzer H., et al. :1981 Astron. Ap.,171,41

Osterbrock D.E.: 1981, Ap. J. 249, 462

Petrosian A.S., et al. :1979, Astrophysics 15, 250)

Shuder J.M., Osterbrock D.E.: 1981, Ap. J. 250, 55

Veilleux S., Osterbrock D.E.: 1987, Ap.J. Suppl. 63, 295

Whittle M., et al. : 1988, Ap.J. 326,125 